\title{
Connectivity restoration and amelioration in wireless ad-hoc networks: A practical solution
}

\author{
Christos Katsikiotis, Dimitrios Zorbas, Periklis Chatzimisios \\ dimitrios.zormpas@inria.fr
}

\begin{abstract}
Connectivity restoration after a node failure is one of the major issues in wireless ad-hoc networks. In particular, failures can lead to a network partitioning and a huge loss of information. Therefore, a fast mechanism is needed to heal the network between the partitions. In this paper, we consider the scenario where an intermediate node failures and a mobile system is moving autonomously to restore connectivity and provide the best service. We propose a fast connectivity restoration algorithm that is based only on local information. We implement our solution on a real robotic platform and we present some experimental results using a simple case scenario.
\end{abstract}

\section{Introduction}

Recently, the use of wireless ad-hoc networks exhibits an exponential increase in different environments and applications. However, one of the vulnerabilities of wireless ad-hoc networks is the appearance of connectivity failures between the network participants that can lead to network partitions and, thus, loss of information. This kind of failures may happen due to the appearance of corrupted nodes or due to their energy exhaustion [13] [11] [18].

The purpose of this paper is to present a practical solution to tackle the aforementioned problem. Specifically, we assume that mobile routers are capable of detecting failures and restoring connectivity between one or more routing points. Once a failure has been detected, the mobile router's first objective is to look for neighboring nodes by moving in the periphery of the routing point and stop when the best link quality has been achieved. The router's second objective is to balance the throughput between the two sides in order to avoid bottlenecks. Depending on the application, the mobile router can act as permanent or a temporary solution which restores all or part of the network services, until the corrupted nodes are replaced and the network returns to its normal operation.

The implementation of such a solution poses several challenges since (a) the successor nodes' position is not known in advance, (b) the signal quality is not stable due to environmental changes, and (c) the connectivity restoration must 
be done very soon without delays. Hence, placing the mobile routers is a fairly complex node deployment problem and constitutes the main focus of the paper.

The contributions of our work are:

- we propose a localized solution, called Connectivity Restoration and aMelioration $(C R A M)$, to restore connectivity between two network partitions;

- we implement our solution on a real robotic platform taking into account both restoration and bandwidth amelioration parameters;

- we evaluate our approach creating a simple case scenario and present our findings.

The remainder of the paper is structured as follows. In Section 2, we discuss the related work in the areas of connectivity restoration and link quality amelioration. In Section 3 we present the problem and in Section 4 its practical solution. In Section 5, we evaluate our approach and present some experimental results. Finally, Section 6 concludes the paper and lists our future work.

\section{Related Work}

The problem of failure nodes resulting limited connectivity in wireless ad-hoc networks has recently received much attention in the literature $[1,2,3,8]$.

Restoring connectivity by moving some of the network nodes is a research path that has been proposed by many works. In [1], the authors propose the DARA algorithm that moves nodes according to their number of neighbors in order to replace the failed ones. In [2], when a node has become inactive and, thus, causing network partitions, it is been suggested that federating the partitions could restore the connectivity by using the previous route information and moving the nodes. In [3], the authors suggest similar approaches that implement federation on disjoint parts of the network to restore connectivity. Finally, a distributed approach based on battery triggered events is proposed in [12]. Robots are used to replace failure nodes using other nodes in the neighborhood. The connectivity is held by computing a connected dominating set.

Also, remarkable research has been done on finding the ideal position between two nodes to maximize the performance of exchanging data between them. Finding such a position is of a great importance, since the deployment of an extra mobile routing point resolves connectivity. Works of $[15,14]$ recommend the use of the APA algorithm. APA estimates the link quality using either the Round-Trip Time or the Transmission Rate or the Received Signal Strength. The calculations are done locally by an autonomous mobile router. A faster version of APA is proposed in [16] and it is applicable when the distance between the two nodes is known. APA and Fast-APA are evaluated through simulation.

Work of [7] evaluates the implication of the signal strength and data throughput in the estimation of link quality. The signal strength is suggested as a good measure of potential connectivity, while data throughput should be used for ensuring minimum actual data transmission rate. The authors of [5] and [6] 
conclude that the link estimation is more efficient when several link quality metrics are combined together. However, as explained in survey of Baccour et al. [4], high overhead (memory footprint, computation time) are still open problems, when several link properties are used.

Finally, some works focus on restoring connectivity in disjointed networks $([17,10,9])$, by trying to find the smallest number of deployable nodes using steiner points to repair the disruption of network connectivity. The authors deal with the problem of finding the minimum count and the position of relay nodes. The problem is NP-hard and hence they propose the use of heuristics. This type of solutions are centralized.

\section{The robot redeployment problem}

This paper addresses the problem of network failures which may occur between multiple static stations and cause loss of information. In case of a failure we assume that mobile routers (robots) restore the connectivity between two or more routing points by creating an alternative route between the them.

In this section we model a case scenario which includes a set of stationary nodes that are connected in an ad-hoc network. At some unspecified moment of time, an intermediate node becomes inactive causing a communication interrupt between the rest of the stations. We define a redeployment problem where a mobile routing system is responsible of restoring connectivity providing the best possible network service at the same time.

In more detail, the objectives of the mobile router are to:

- restore connectivity as soon as possible,

- ensure a link quality (between both stations) which is in the worst case equal to $h$,

- avoid network bottlenecks by balancing the throughput of the links between the two sides.

\section{The CRAM solution}

The CRAM algorithm (Connectivity Restoration And aMelioration) is introduced in order to solve the previously aforementioned node deployment problem. CRAM is a localised algorithm which takes into account only neighboring information in order to compute its movement. No global information about the position of the stations is required. The only assumption that CRAM makes is that the mobile router is located close to one of the 1-hop neighbors of the corrupted node when the failure happens. Figure 1 depicts an example of the positions of the devices before the failure.

The algorithm is divided in two phases. During the first phase, a node discovery process is taking place in order to identify the location of the next hop and restore connectivity. Since the next hop is detected, the second phase 


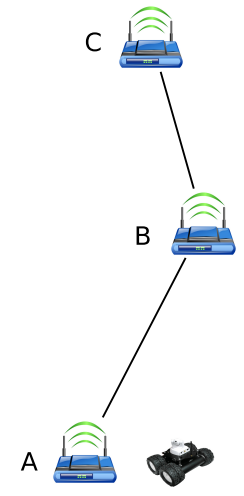

Figure 1: Initial network layout.

deals with the amelioration of the link quality between the robot and nodes A and $\mathrm{C}$.

The discovery phase includes several small movements and after each movement the link quality between the router and the routing points $\mathrm{A}$ and $\mathrm{C}$ is measured. The robot's first movement is to move away from point A traveling a straight line distance equal to $r$. This distance must not be higher than the distance between $\mathrm{A}-\mathrm{C}$. The direction of the movement is random.

The robot makes a number of small steps following an almost circular movement around A with radius $r$. After each movement, it measures the link quality between itself and routing points A and C. The RSSI value is used as a metric of the measurements and it varies between -100 and $0 \mathrm{dBm}$. When no connection exists, the robot considers a RSSI value equal to -100 . Only values above $h$ are considered acceptable, while the rest of the values are discarded. An average value of ten measurements for each link with an interval of $d t$ seconds is considered.

The robot's circular movement can be done either clockwise or counterclockwise. The computation of the next position at which the robot will stop to measure again the link quality is given by Formula (1).

$$
(x, y)=(r \cos (i \theta), r \sin (i \theta)),
$$

where $i$ is the number of movements and $\theta$ is the angle which corresponds to an arc whose chord length (step) is equal to $d$ meters (see Figure 2).

The robot follows a set of rules in order to decide where to move and when to stop moving. First of all, the robot's circular movement continues as far as the RSSI value between the robot and node $\mathrm{C}$ is below $h$. Once the robot computes an average RSSI value above the threshold, it keeps moving, storing in its memory the maximum RSSI value and the position where it was achieved. If during the next movements, the link quality falls bellow a threshold $p$, the robot returns to the position where the maximum RSSI values was captured. $p$ is defined as the signal attenuation between the current position and the position 


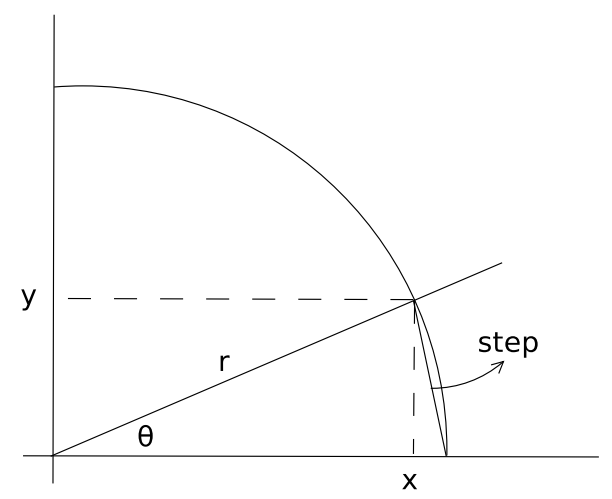

Figure 2: Computation of the router's next position.

where the best signal quality was measured.

If no position is found during a full circle with signal level above $h$, then the robot gives up its effort reporting an error. The maximum number of movements $\max _{-} i$ during a full round can be calculated combining the equations in (2):

$$
\max \_i=\left\lceil\frac{360}{s}\right\rceil, \quad s=\frac{\theta}{180} \pi r, \quad \theta=2 \arcsin \frac{d}{2 r},
$$

where $s$ is the length of the arc and $\theta$ is the angle in degrees.

The maximum total time of the discovery phase is given by Formula (3).

$$
\text { max_time }=\frac{r}{V}+\max \_i\left(\frac{d}{V}+9 d t\right),
$$

where $V$ is the speed of the robot.

At this point the discovery phase has been terminated, giving way to the amelioration phase. This second phase is needed since the robot may be very close or distant to one of the routing points causing link instability. In order to avoid this problem, we use the link amelioration algorithm presented in [14]. The algorithm balances a link quality metric between two sides by applying a step-by-step moving similarly to the circular movement we presented before. The robot's movement is made on a straight line defined by its current and A's position.

According to this algorithm, the mobile router compares the two signals and moves towards to the station with the weakest one. The robot continues moving until the difference between the signals is below a predefined threshold $q$. In order to avoid network bottlenecks, we use the throughput of the communication channel as metric of our measurements.

\section{Evaluation \& Discussion of the Results}

In this section we evaluate the performance of CRAM by performing a real experiment with three stations and a mobile router. Although, the same ex- 
periment has been repeated several times by using different configurations and values, in this section one of the most representative scenarios is employed.

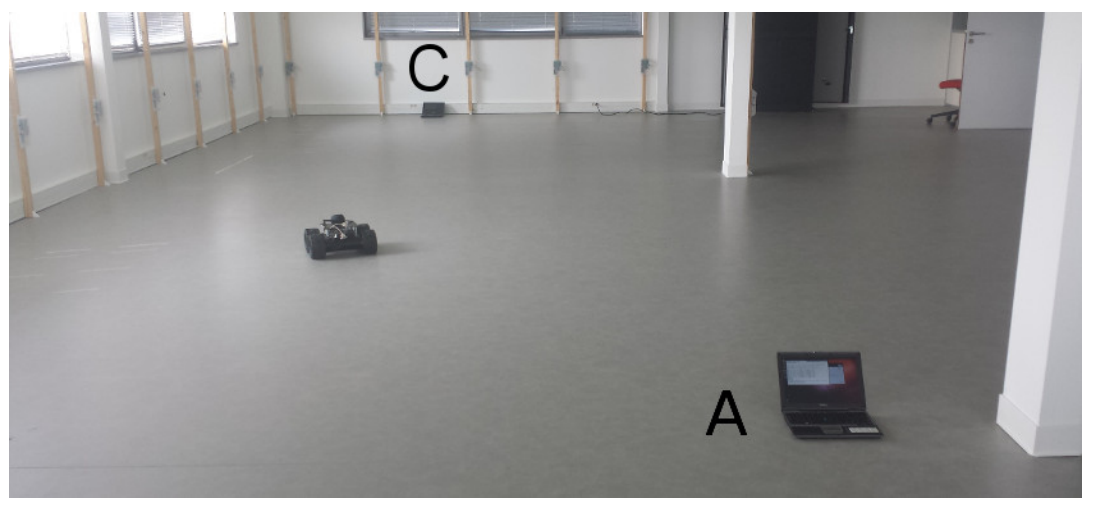

Figure 3: The experimental setup.

The experiment consists of three stationary nodes that are part of an adhoc network and a mobile robot $^{1}$ which has the ability to move onward and backward as well as to rotate in different angles. All the participants used IEEE $802.11 \mathrm{~g}$ compatible wireless network cards. The experimental setup is illustrated in Figure 3. In this picture we have excluded the failure node B. The two other stations are placed 12 meters away to each other, the radius for the circular movement is predefined equal to 2 meters and the step $d$ is 0.78 meters. The step of the straight movement is set equal to 1 meter. We also consider a time interval equal to 0.1 seconds between the ten RSSI measurements as well as $h, p$ and $q$ thresholds equal to $-65 \mathrm{dBm}, 5 \mathrm{dBm}$ and $3 \mathrm{Mbps}$, respectively. The angle between stations $\mathrm{A}$ and $\mathrm{C}$ is set equal to 120 degrees. The robot has no knowledge about the position of station $\mathrm{C}$.

We assume that the connectivity between the stations is lost (it has fallen bellow $h$ ) and the robot starts moving at that moment of time. In the following lines we present the experimental results derived by the robot's measurements.

As we have already mentioned, CRAM is executed in two phases. During the first phase the connectivity is restored, while in the second phase, the throughput between the two routing points and the robot is improved. Figure 4 presents the positions of the stations as well as the robot's intermediate and final positions. The intermediate position denotes the robot's location after the first phase of the algorithm.

Figure 5 illustrates the robot's movement and the corresponding RSSI values of each step. During the first steps, there is no connection between the router and station $\mathrm{C}$ or the link quality is poor (below $h$ ), thus, the RSSI value is considered equal to -100 . The router detects C's signal after the third step, while the maximum value is measured about 120 degrees away from station A. The signal level at that position is stored and the robot continues its movement.

\footnotetext{
${ }^{1} \mathrm{http}: / /$ www.wifibot.com/
} 


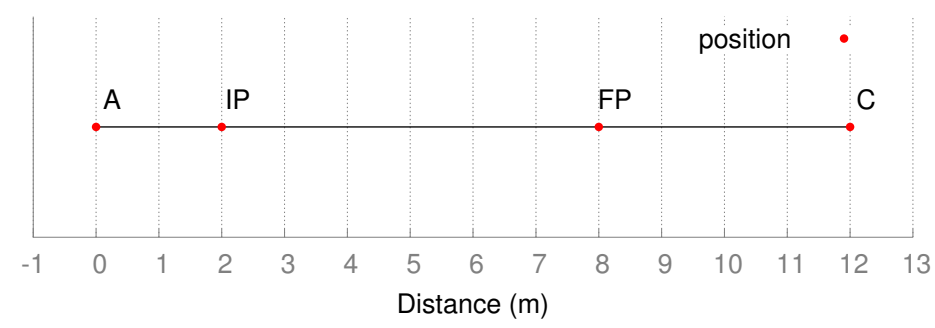

Figure 4: The distance between the robot and stations A, C throughout the deployment (IP: intermediate position after the circular movement, FP: final position)

Since after the next step the link quality falls bellow the predefined threshold $p$, the robot returns to the position where the best signal was captured.

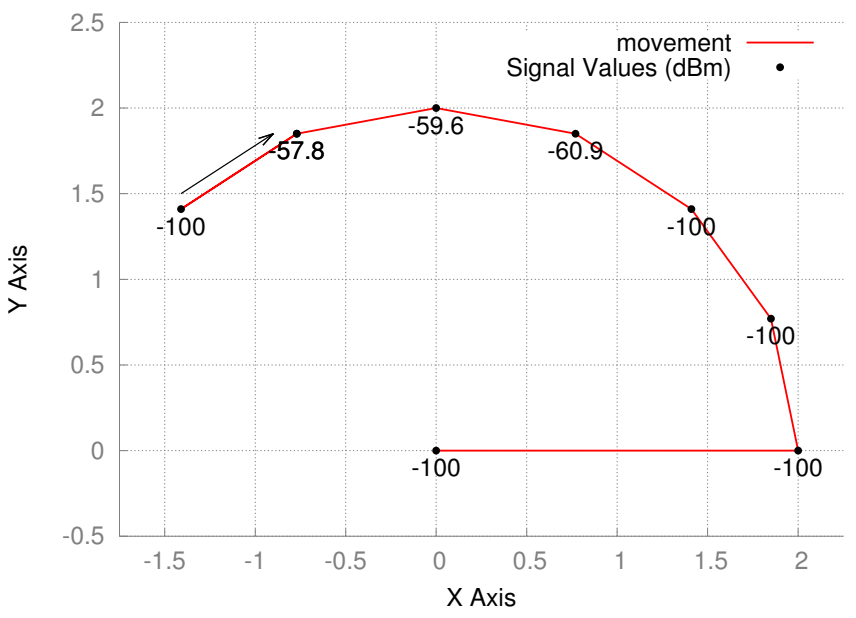

Figure 5: The position of robot each time

Figure 6 depicts the bandwidth level between the mobile router and the two routing points for the second phase movement. The results show that the movement was completed in eight steps. The robot stops when the two values are below the specified threshold $q$. We can observe a great improvement in terms of the achieved bandwidth between the initial and the final position.

Finally, the robot's traveling distance during the second phase of the deployment is illustrated in Figure 7. The figure shows that the robot moved 7 times towards the station $\mathrm{C}$ and one time backwards in order to find the best measurement. The distance is measured from the point the previous phase was terminated. 


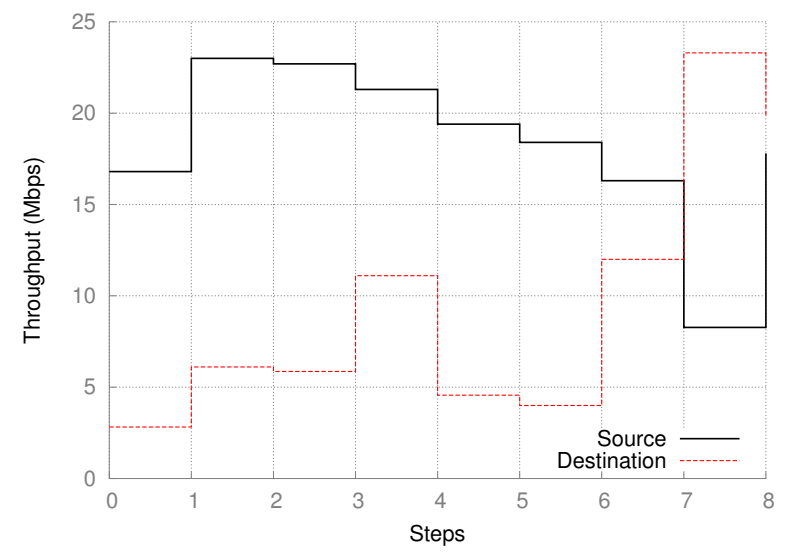

Figure 6: The signal levels between the router and stations A, C.

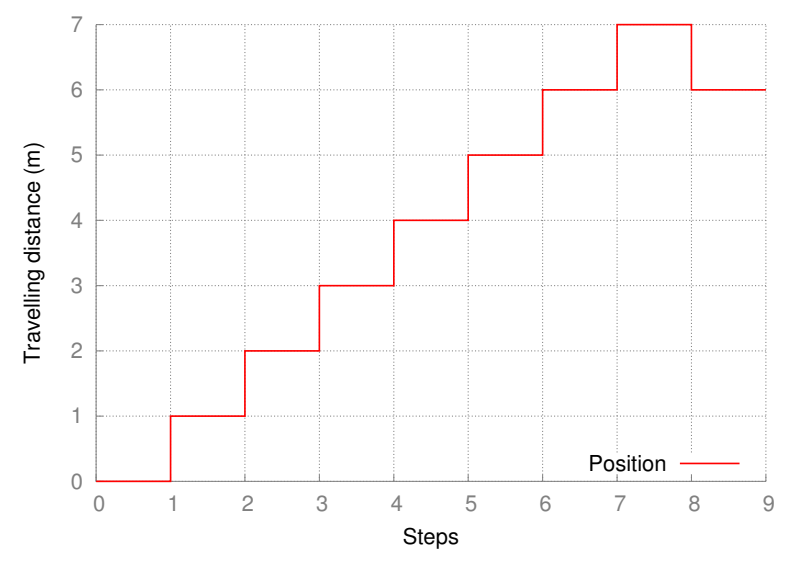

Figure 7: The robot's traveling distance for each step of its second phase movement.

\section{Conclusion \& Possible Future Work}

In this paper, we have proposed an efficient algorithm to restore connectivity between network partitions in a wireless ad-hoc network using another node as an alternative route. The solution is based on local information and its effectiveness is evaluated through experimentation. The results of the experiment are very encouraging and give the confidence that the connectivity between two nodes can be easily restored and ameliorated, thus, we are able to restore the network performance, without the need of robot to take many steps and consume big amounts of energy.

Our future work aims at the extension of the CRAM algorithm by performing more complicated and realistic scenarios. In particular, we investigate the 
use of CRAM in a multiple routing point layout, where the robot has to restore connectivity between more than two routing points. Furthermore, we are interested in developing a multi-robot system when failures occur further than one hop. In this case, there is a necessity of deploying more than one robot to restore connectivity. Finally, a multi-failure scenario is going to be considered. In this scenario, failures occur in more than one node and the robot is responsible to evaluate the importance of each partition of the network and resolve connectivity by an order of importance.

\section{Acknowledgments}

This work is partially supported a grant from CPER Nord-Pas-de-Calais FEDER CIA.

\section{References}

[1] AA Abbasi, K. Akkaya, and M. Younis. A distributed connectivity restoration algorithm in wireless sensor and actor networks. In Local Computer Networks, 200\%. LCN 2007. 32nd IEEE Conference on, pages 496-503, Oct 2007.

[2] Kemal Akkaya, Izzet F. Senturk, and Shanthi Vemulapalli. Handling largescale node failures in mobile sensor/robot networks. Journal of Network and Computer Applications, 36(1):195 - 210, 2013.

[3] F.M. Al-Turjman, H.S. Hassanein, and M. Ibnkahla. Optimized relay placement to federate wireless sensor networks in environmental applications. In Wireless Communications and Mobile Computing Conference (IWCMC), 2011 7th International, pages 2040-2045, July 2011.

[4] Nouha Baccour, Anis Koubâa, Luca Mottola, Marco Antonio Zúñiga, Habib Youssef, Carlo Alberto Boano, and Mário Alves. Radio link quality estimation in wireless sensor networks: A survey. ACM Trans. Sen. Netw., 8(4):34:1-34:33, September 2012.

[5] Nouha Baccour, Anis Kouba, Habib Youssef, Maissa Ben Jama, Denis do Rosrio, Mrio Alves, and LeandroB. Becker. F-lqe: A fuzzy link quality estimator for wireless sensor networks. In JorgeS Silva, Bhaskar Krishnamachari, and Fernando Boavida, editors, Wireless Sensor Networks, volume 5970 of Lecture Notes in Computer Science, pages 240-255. Springer Berlin Heidelberg, 2010.

[6] C.A Boano, M.A Zuiga, T. Voigt, A Willig, and K. Romer. The triangle metric: Fast link quality estimation for mobile wireless sensor networks. In Computer Communications and Networks (ICCCN), 2010 Proceedings of 19th International Conference on, pages 1-7, Aug 2010. 
[7] M. Ani Hsieh, Anthony Cowley, Vijay Kumar, and Camillo J. Taylor. Maintaining network connectivity and performance in robot teams. Journal of Field Robotics, 25(1-2):111-131, 2008.

[8] Kyu-Han Kim, K.G. Shin, and D. Niculescu. Mobile autonomous router system for dynamic (re)formation of wireless relay networks. Mobile Computing, IEEE Transactions on, 12(9):1828-1841, Sept 2013.

[9] Sookyoung Lee and Mohamed Younis. Recovery from multiple simultaneous failures in wireless sensor networks using minimum steiner tree. Journal of Parallel and Distributed Computing, 70(5):525 - 536, 2010.

[10] Sookyoung Lee and Mohamed Younis. Optimized relay node placement for connecting disjoint wireless sensor networks. Computer Networks, $56(12): 2788-2804,2012$.

[11] Wenjia Li, A Joshi, and T. Finin. Coping with node misbehaviors in ad hoc networks: A multi-dimensional trust management approach. In Mobile Data Management (MDM), 2010 Eleventh International Conference on, pages 85-94, May 2010.

[12] Kalypso Magklara, Dimitrios Zorbas, and Tahiry Razafindralambo. Node discovery and replacement using mobile robot. In Jun Zheng, Nathalie Mitton, Jun Li, and Pascal Lorenz, editors, Ad Hoc Networks, volume 111 of Lecture Notes of the Institute for Computer Sciences, Social Informatics and Telecommunications Engineering, pages 59-71. Springer Berlin Heidelberg, 2013.

[13] Sergio Marti, T. J. Giuli, Kevin Lai, and Mary Baker. Mitigating routing misbehavior in mobile ad hoc networks. In Proceedings of the 6th Annual International Conference on Mobile Computing and Networking, MobiCom '00, pages 255-265, New York, NY, USA, 2000. ACM.

[14] Karen Miranda, Enrico Natalizio, and Tahiry Razafindralambo. Adaptive Deployment Scheme for Mobile Relays in Substitution Networks. International Journal of Distributed Sensor Networks (IJDSN), 2012, 2012.

[15] Karen Miranda, Enrico Natalizio, Tahiry Razafindralambo, and Antonella Molinaro. Adaptive router deployment for multimedia services in mobile pervasive environments. In Proceedings of the Work in Progress session at PerCom (WIP of PerCom), pages 471-474, Lugano, Switzerland, March 19-23 2012.

[16] Jean Razafimandimby, Karen Miranda, Dimitrios Zorbas, and Tahiry Razafindralambo. Fast and reliable robot deployment for substitution networks. In Proceedings of the 10th ACM Symposium on Performance Evaluation of Wireless Ad Hoc, Sensor, and Ubiquitous Networks (PE-WASUN), pages 17-23, Barcelona, Spain, nov 2013. 
[17] Fatih Senel and Mohamed Younis. Relay node placement in structurally damaged wireless sensor networks via triangular steiner tree approximation. Computer Communications, 34(16):1932 - 1941, 2011.

[18] Vikram Srinivasan, Pavan Nuggehalli, C.F. Chiasserini, and R.R. Rao. Cooperation in wireless ad hoc networks. In INFOCOM 2003. Twenty-Second Annual Joint Conference of the IEEE Computer and Communications. IEEE Societies, volume 2, pages 808-817 vol.2, March 2003. 